\title{
STUDY OF THE EFFECT OF SODIUM DODECYL SULFATE (SDS) AND ACRIDINE ORANGE ON THE ISOLATION OF Plasmid ANd Antimicrobial Resistance PatTern OF CLINICAL ISOLATES OF KLEBSIELLA SP
}

\author{
Mohammad Shahriar ${ }^{1 *}$, Shumaila Mawla ${ }^{1}$, Mohiuddin Ahmed Bhuiyan ${ }^{1}$ and Mahboob \\ Hossain $^{2}$ \\ Department of Pharmacy, The University of Asia Pacific, Dhanmondi, Dhaka, Bangladesh ${ }^{1}$ \\ Department of Microbiology, BRAC University, Mohakhali, Dhaka, Bangladesh ${ }^{2}$ \\ E-mail: shahriar_12@yahoo.com
}

\begin{abstract}
For a six months period a total of 25 Klebisiella sp. samples collected from different diagnostic centers were tested for their antimicrobial susceptibility pattern against 16 antimicrobials. All isolates under study showed resistance against cephalexin, cephradine, ciporfloxacin, cloxacillin, erythromycin, oxacillin, rifampicin and tetracycline. Most of the isolates were resistant to amoxicillin (92\%), vancomycin (96\%), neomycin (84\%) and chloramphenicol (76\%). Least number of isolates showed resistance to tetracycline (36\%), ceftriaxone (40\%) and gentamycin (56\%), respectively. Out of the 25 isolates, 7 multidrug resistant isolates were selected and they were grown in varying concentrations of Sodium Dodecyl Sulfate (SDS) in Luria broth and their plasmid profile was analysed. Distinct bands of plasmid were observed when the multidrug resistant strains of Klebsiella sp. were grown in 5\% SDS and acridine orange $(10 \mu \mathrm{g} / \mathrm{ml})$ in Luria broth. On being treated with 5\% SDS and 5\% SDS along with acridine orange, one isolate (isolate no. 3) developed sensitivity against ceftriaxone, ciprofloxacin, co-trimoxazole, gentamycin and neomycin.
\end{abstract}

Keywords: Klebsiella sp., plasmid, antimicrobial susceptibility, multidrug resistan and, sodium dodecyl sulfate (SDS)

\section{Introduction}

In recent years, Klebsiella have become important pathogens in nosocomial infections [1]. Klebsiella $s p$. is well known to most clinicians as a cause of community-acquired bacterial pneumonia, occurring particularly in chronic alcoholics and showing characteristic radiographic abnormalities due to a severe pyogenic infection. They possess a polysaccharide capsule, which is the main determinant of their pathogenisity. The capsule is composed of complex acidic polysaccharides. Its massive layer protects the bacterium from phagocytosis by polymorphonuclear granulocytes [2].

Inappropriate antimicrobial treatment and overuse of antimicrobials have been a contributing factor to the emergence of resistant bacteria. Antimicrobials are frequently prescribed for the indications in which their use is not warranted, an incorrect or sub-optimal antibiotic is prescribed or in some cases for infections likely to resolve without treatment [3]. Resistance to a single drug can spread rapidly through a bacterial population. Increasing antimicrobial resistance has been reported for Klebsiella sp. The microorganisms remain highly susceptible to most antimicrobials [4]; only one isolate was resistant to third-generation cephalosporins.

Plasmids may carry genes that provide resistance to naturally occurring antibiotics in a competitive environmental niche, or alternatively the proteins produced may act as toxins under similar circumstances. Cell lysis is vital for the extraction of DNA, RNA and Proteins from cells. Therefore, it is needed in most cell biology techniques and even molecular biology techniques.

Objectives behind this particular study are determination of which antimicrobials the 
microorganism is resistant to, determination of the correlation of antimicrobial resistance pattern with their plasmid profile, determination of the effect of SDS (sodium dodecyl sulphate) and acridine orange on plasmid isolation and antimicrobial resistance pattern of multi-drug resistant clinical isolates of Klebsiella sp. and as a guide to know which antibiotics the microorganism is sensitive for those patients suffering from infections caused by Klebsiella sp.

\section{Materials and Methods 2.1 Source of Sample Collection}

Twenty five isolates of Klebsiella sp. were collected from laboratories of different diagnostic centers during six months study period. The samples were collected from pathological specimen. For the purpose, slants of McConkey agar were prepared in test tubes for collecting the samples and incubated them at $37^{\circ} \mathrm{C}$ for $18 \mathrm{hrs}$. Red colony grew in the media depicting the presence of gram negative bacteria.

\subsection{Antimicrobial Susceptibility Test}

The antimicrobial susceptibility pattern of Klebsiella $s p$. to 17 antibiotics namely amoxicillin, Am (30 mcg/disc), ceftriaxone, Ci (30 mcg/disc), cephalexin, Cp (30mcg/disc), cephradine, CV (25mcg/disc), chloramphenicol, C (30mcg/disc), ciprofloxacin Cf (5mcg/disc), cloxacillin CX (1mcg/disc), co-trimoxazole, Co (25mcg/disc), erythromycin, E (15mcg/disc), gentamycin G (10mcg/disc), neomycin N (30 mcg/disc), oxacillin OX (1 mcg/disc), penicillin G P (10mcg/disc), rifampicin $R$ (5mcg/disc), tetracycline $\mathrm{T}$ (30mcg/disc) and vancomycin VA (30 mcg/disc) was determined by disc diffusion Kirby Bauer method [5,6]. All tests were performed in Mueller-Hinton Agar plates.

From the first antibiogram, 7 multidrug resistant samples were selected. Each of 7 samples of Klebsiella sp. was treated with $0.1 \%, 1 \%$ and $5 \%$ SDS respectively. Antibiotic susceptibility test for these 7 samples was carried out in absence of SDS and each of those isolates were treated with $0.1 \%, 1 \%$ and $5 \%$ SDS by disc diffusion Kirby Bauer method [5,6] using the same 17 antimicrobials. The plasmid profile analysis of the above samples was done using gel electrophoresis.

\subsection{Plasmid Isolation}

To isolate plasmid DNA from bacteria, the alkaline lysis procedure is used miniprep [7]. The above method was carried for liquid cultures of the multi-drug resistant and sensitive Klebsiella Sp. isolates containing 0.1\%, $1 \%$ and $5 \%$ SDS (Sodium Dodecyl Sulphate) respectively and the result of their plasmid isolation was observed under UV light.

\subsection{Separation of Plasmid DNA By Agarose Gel Electrophoresis}

Agarose gel electrophoresis [7] method was used to separate Plasmid DNA obtained from the isolates. The plasmid DNA was subjected to electrophoresis in 1.5\% Agarose in TBE buffer for about 90 minutes to allow the loading dye in each sample to migrate near the bottom of the gel. The gel was viewed on an ultraviolet (UV) light box (short wave, ultraviolet products, Inc., San Gabriel, California, USA, 254nm).The photographs were taken under UV illumination using Sony cyber shot 5.1 megapixel, USA.

\section{Results}

The culture sensitivity pattern of Klebsiella sp. isolates under study to different antimicrobials isolated from different pathological specimens demonetrated that most isolates showed resistance to some commonly used antimicrobials such as amoxicillin, cephalexin, cephradine, chloramphenicol, ciprofloxacin, cloxacillin, co-trimoxazole, erythromycin, neomycin, oxacillin, penicillin $\mathrm{G}$, rifampicin and vancomycin (as shown in Figure 1). Isolates obtained from urine were found to be resistant to antimicrobials. All isolates showed resistance to cephalexin, cephradine, ciporfloxacin, cloxacillin, erythromycin, oxacillin, rifampicin and tetracycline respectively. Least number of isolates showed resistance to tetracycline (36\%) and ceftriaxone (40\%) and gentamycin (56\%) respectively. From the study of antimicrobial resistance pattern, 7 multidrug resistant isolates were selected (C-437 URINE KLEB, KLEB C014, C-282 URINE KLEB, 512 KLEB, C-579 URINE KLEB, 415 URINE KLEB and M-204 URINE KLEB ). 
Sample 415 Urine Kleb, which is a multidrug resistant Klebsiella sp. isolate, developed sensitivity for ceftriaxone, ciprofloxacin, cotrimoxazole, gentamycin and neomycin respectively on treatment with 5\% SDS and 5\% SDS along with acridine orange $(10 \mu \mathrm{g} / \mathrm{ml})$. Isolate C-437 Urine Kleb developed resistance for ceftriaxone on being cultured in 5\% SDS and acridine orange in Luria broth.

Table 1 shows the trend of culture sensitivity of treated and untreated Klebsiella sp. isolates with $5 \%$ SDS and acridine orange to different antimicrobials. It was found that all the 5\% SDS and acridine orange treated and untreated isolates were resistant to amoxycillin, cephalexin, cephradine, and cloxacillin.

In the present study SDS was used because it is a curing agent and hence it leads to the formation of pores in the bacterial cell wall through which the plasmid come out of the cell which was also indicated in the gel electrophoresis [8]. In the plasmid analysis of the Klebsiella $s p$. isolates grown in 5\% SDS in Luria broth as shown above in Figure 2, plasmid was isolated from the multidrug resistant isolates such as KLEB C-014 (1s), C-282 URINE KLEB (2s), 415 URINE KLEB (3s), C-579 KLEB (4s), 512 KLEB (6s) and KLEB C-36 (7s). Plasmids were also found in the isolates KLEB C-014 (1As), C-282 URINE KLEB (2As), C-579 KLEB (4As) and KLEB C36 (7As) respectively when grown in 5\% SDS and acridine orange in Luria broth. For the multidrug resistant isolates, plasmids appeared to be of similar size which may indicate that all cells contained plasmid. But based on this observation we cannot confirm that the drug resistant genes are present in the plasmids. Klebsiella sp. isolate 415 URINE KLEB developed sensitivity for ceftriaxone, ciprofloxacin, co-trimoxazole, gentamycin and neomycin respectively. It appears from this finding (as shown in Figure 2) the isolate 415 URINE KLEB lost its plasmids when grown in the presence of SDS and acridine orange. So it appears that ceftriaxone, ciprofloxacin, co-trimoxazole, gentamycin and neomycin resistant genes may be present in plasmids. As a result, it can be concluded that plasmid gene may or may not be responsible for developing multi-drug resistance for the isolate 415 URINE KLEB. However, further study is necessary to confirm this issue because in case of other isolates presence or absence of plasmid has no relation with ceftriaxone, ciprofloxacin, cotrimoxazole, gentamycin and neomycin respectively.

Presence of plasmids were observed in Klebsiella sp. isolates, namely KLEB C-014, C579 KLEB, and 512 KLEB, although there was no change in their antimicrobial susceptibility pattern on being grown in 5\% SDS (sodium dodecyl sulfate). There was no significant change in result of plasmid profile analysis on growing the Klebsiella $s p$. isolates in presence of acridine orange $(10 \mu \mathrm{g} / \mathrm{ml})$ along with 5\%SDS.

\section{Discussion}

Drug resistance in microorganisms is a predictable and perhaps inescapable response to the use of antimicrobial agent. It can arise from the selection of resistant strains among naturally susceptible species or from the ingress of new strains of naturally resistant species [9]. The extent of use of particular agents in a given environment dictates the rate at which resistance arises among microbial populations. In the last several years, the question has arisen whether it is necessary to determine if each isolated Klebsiella sp. strain is an ESBL producer [10]. The answer depends on the epidemiologic situation of a country or a hospital, but it should definitely be positive if a high percentage of ceftazidime-resistant strains is to be expected. To date, two diagnostic tests have been most commonly used for the detection of such isolates. In the double-disc synergy test, a disc of clavulanic acid and a disc of an extendedspectrum cephalosporin such as ceftazidime are placed close together on an agar surface inoculated with the test organism [10].

In the present study, Klebsiella sp. isolates were collected from different pathological specimens and seventeen different antimicrobial agents were used to test the susceptibility. Most of the Klebsiella isolates of this study showed resistance to the first line antibiotics that are commonly prescribed by the physicians. Significant isolates showed resistance to some commonly used antimicrobials. Isolates obtained from urine were found to be resistant to antimicrobials. All isolates showed resistance to 


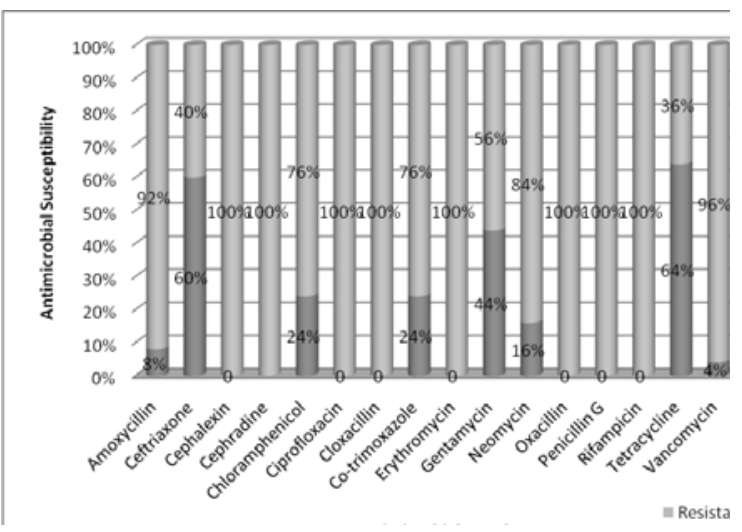

Fig. 1: Antimicrobial Susceptibility pattern of isolates of Klebsiella sp.

cephalexin, cephradine, ciporfloxacin, cloxacillin, erythromycin, oxacillin, rifampicin and tetracycline respectively (Fig.1). Lowest number of isolates showed resistance to tetracycline (36\%) and ceftriaxone (40\%) and gentamycin (56\%) respectively.

A change that was observed in our present study is that plasmid isolation was more effective when the isolates were grown in SDS (sodium dodecyl sulfate). The results of plasmid analysis varied with concentrations of SDS in Luria broth (Table 1). No specific change was observed when plasmid isolation (Fig.2) was done to Klebsiella sp. isolates grown in SDS and acridine orange $(10 \mu \mathrm{g} / \mathrm{ml})$. An attempt was taken to carry out plasmid curing but it was rendered unsuccessful in our present study.

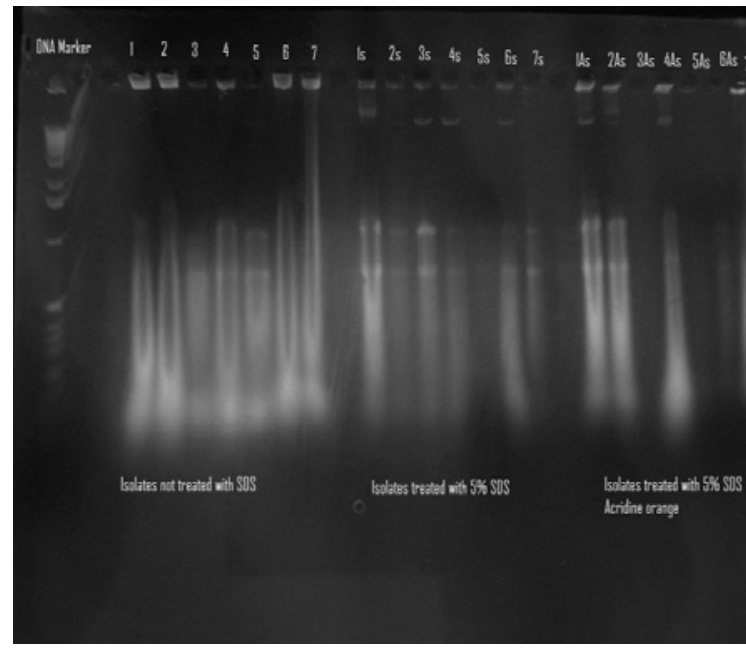

Fig. 2: Electrophoretic patterns showing plasmid DNA in multidrug resistant isolates of Klebsiella sp. grown in $5 \%$ SDS \& acridine orange $(10 \mu \mathrm{g} / \mathrm{ml})$ in Luria broth
Resistance factors, particularly those carried on mobile elements, can spread rapidly within human and animal populations. Multidrugresistant pathogens travel not only locally but also globally, with newly introduced pathogens spreading rapidly in susceptible hosts. Antibiotic resistance is frequently determined by genetic information of plasmid origin [11]. The correlation between antibiotic resistance and plasmid profile may indicate that the genetic information is plasmid borne. Initially, there was a tendency to assume that antibiotic resistance genes appeared only after antibiotics began to be used widely in medicine. However, the genetic diversity within some classes of resistance makes it clear that the genes have been evolving for a much longer time [11].

Widespread antibiotic usage exerts a selective pressure that acts as a driving force in the development of antibiotic resistance. The association between increased rates of antimicrobial use and resistance has been documented for nosocomial infections as well as for resistant community acquired infections [12]. As resistance develops to "first-line" antibiotics, therapy with new, broader spectrum, more expensive antibiotics increases, but is followed by development of resistance to the new class of drugs.

\section{Conclusion}

In the present study, it is observed that antimicrobial resistance to Klebsiella $s p$. has been increased from the previous studies carried out $[13,14]$. To avoid these anomalies, prescription of antimicrobials by quack should be prohibited, physicians should not prescribe without pathological investigation as well as government doctors, pharmacists, patients and all health related personnel should be alert and conscious to ensure rational use of antimicrobials. In present study, the result obtained indicates that there is a correlation between plasmid and antibiotic resistance of Klebsiella $s p$. But further studies are to be carried out in future to confirm this issue. Besides if the gene responsible for multidrug resistance can be located, genetic engineering and further research can be done to prevent Klebsiella sp. from becoming resistant. 
Table 1: Culture Sensitivity Pattern of Klebsiella sp. to different antimicrobials (grown in absence and presence of 5\% SDS and acridine orange in Luria broth)

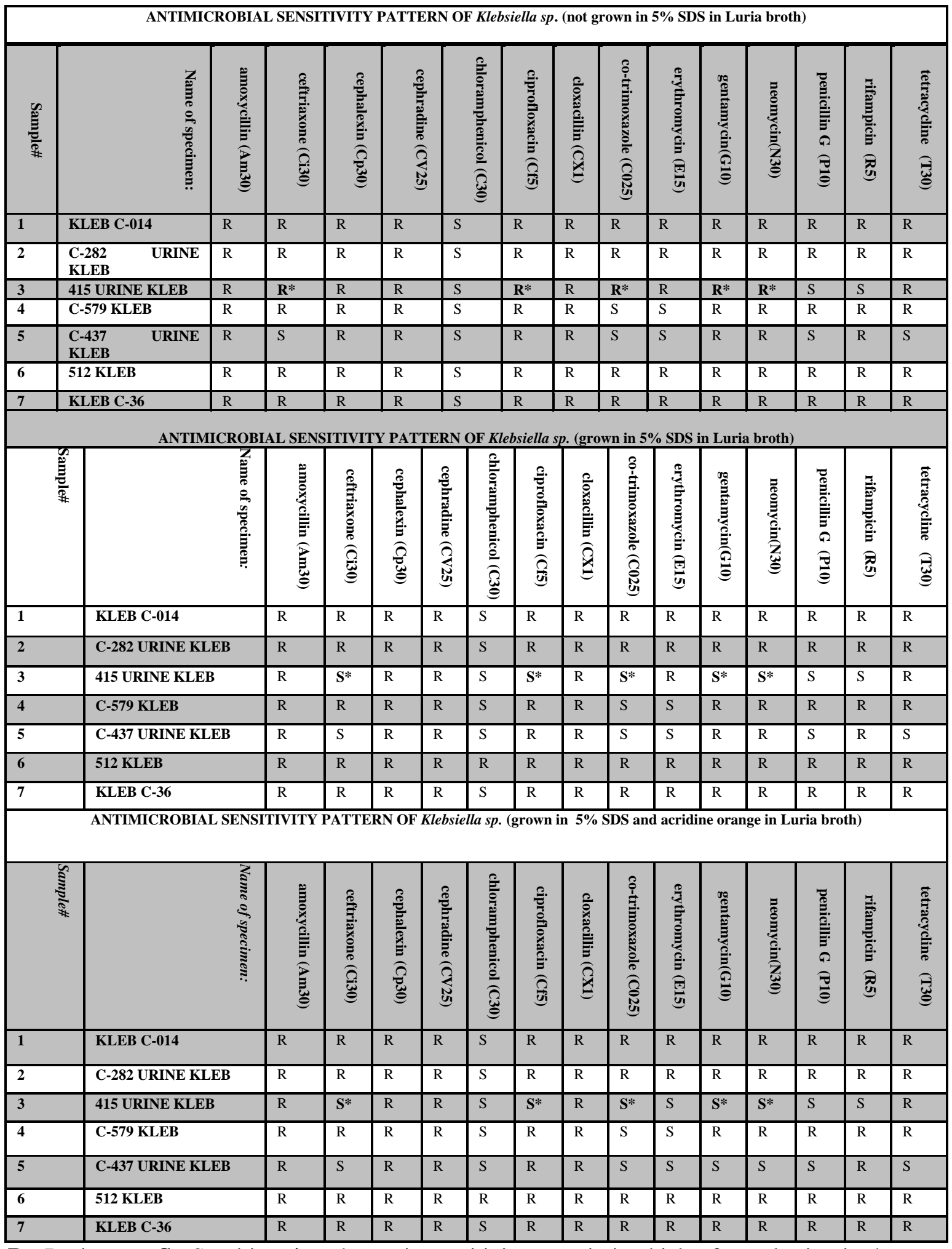

$\mathbf{R}=$ Resistance, $\mathbf{S}=$ Sensitive, $*$ = change in sensitivity to antimicrobials after culturing in the presence of $5 \%$ SDS and acridine orange $(10 \mu \mathrm{g} / \mathrm{ml})$ in Luria broth. 


\section{References}

[1] P. Nordmann, G. Cuzon, T. Naas, “The real threat of Klebsiella pneumoniae carbapenemase-producing bacteria”, Lancet Infect Dis. Vol. 9, 2009, pp. 22836.

[2] Y. R. Chan, J. S. Liu, D. A. Pociask, M. Zheng, T. A. Mietzner, T. Berger, "Lipocalin 2 is required for pulmonary host defense against Klebsiella infection”, J Immunol. Vol. 182, 2009, pp. 49474956.

[3] T. G. Slama, A. Amin, S. A. Brunton et al., "A clinician's guide to the appropriate and accurate use of antibiotics: the Council for Appropriate and Rational Antibiotic Therapy (CARAT) criteria", Am. J. Med., Vol. 118, 2005, pp. 1S-6S.

[4] B. Murray, "New Aspects of antimicrobial resistance and the resulting therapeutic dilemmas", J. Infect Dis, Vol. 63, 1991, pp. 1185.

[5] A. Bauser, W. M. J. Kirby, C. Sheris, and M. Truck, "Antibiotic susceptibility testing by a standard single disc method” ,Am. J. of Clinical Pathology, Vol. 145, 1966, pp. 225-230.

[6] National committee for clinical laboratory standard: Methods for dilution antimicrobial tests for bacteria that grow aerobically, $3^{\text {rd }}$ edn., approved standard. NCCLS, Pennsylvania, Document M7-A3, 1999.

[7] Russell, W. David, and Sambrook, Joseph, Molecular cloning: a laboratory manual, Cold Spring Harbor, N.Y: Cold Spring Harbor Laboratory, 2001.

[8] M. H. Akhter, A. Hussain, S. N. Khan, "Plasmid Mediated Multi-Drug Resistance of Escherichia coli
Isolated from Urinary Tract Infections-A Preliminary Study”, Bangladesh J. Microbiol, Vol. 22, No.1, 2005, pp. 55-58.

[9] C. K. Liam, K. H. Lim, C. M. Wong, “Communityacquired pneumonia in patients requiring hospitalization”, Respirology, Vol. 6, No.3, 2001, pp. 259-64.

[10] R. Podschun, U. Ullmann, "Klebsiella spp. as nosocomial pathogens: epidemiology, taxonomy, typing methods, and pathogenicity factors”, Clin Microbiol Rev., Vol. 11, No. 4, 1998, pp. 589-603.

[11] Tin Tin Myaing, A.A. Saleha, A.K. Arifah and A.R. Raha, "Antibiotic resistance and plasmid carriage among Escherichia coli isolates from chicken meat in Malaysia”, Applications of gene-based technologies for improving animal production and health in developing countries, H. P. Makkar and G. J. Viljoen (eds.), pp 521-527, Printed in the Netherlands, 2005.

[12] J. Sedor, S. G. Mulholland, "Hospital-acquired urinary tract infections associated with the indwelling catheter”, Urol Clin North Am., Vol. 26, No. 4, 1999, pp. 821-8.

[13] J. R. Talbot, D. K. Yamamoto, M. W. Smith and R. J. Seidler, “Antibiotic resistance and its transfer among clinical and non-clinical Klebsiella strains in botanical environment”, Applied Environ Micro., Vol. 39, 1980, pp. 97-104.

[14] S. A. Rasool, A. Ahmed, S. Khan and A. Wahab, "Plasmid borne antibiotic resistance factors among indigenous Klebsiella”, Pak. J Bot., Vol. 35, 2003, pp. 243-248. 